\title{
The concepts of an atom and chemical bond in physics and chemistry: the role of approximations
}

Apart from the theoretically driven evolution of the meaning of some of the important physical and chemical concepts, there is yet another often forgotten source of important problems arising in physics and chemistry. It results from the approximations that are necessary for any application of quantum mechanics to practical problems within these two areas of scientific inquiry. As a side effect of such approximations, the fundamental differences in the understanding of some concepts by a physicist and a chemist may appear. This problem is best demonstrated by the analysis of the concept of an atom and chemical bond as an example.

\section{The picture of atom and chemical bond in classical chemistry and quantum mechanics}

For chemists the atom consists of atomic core with positive effective charge (due to the screening effect of bare nuclear charge from valence electrons by the electrons occupying inner shells) and surrounding valence electrons (i.e. electrons belonging to outermost shells). The valence electrons are entirely responsible for the behavior and properties of particular atom as well as for the 
properties of chemical bonds created with other atoms and these bonds are entities possessing well defined properties like bond length, bond order, bond strength or bond character. Such a view of atoms and bonds is extremely useful and fruitful in analyzing, explaining and predicting the behavior and properties of chemical species in many practical cases. What is more - such „chemical” picture of atoms and bonds prevails in chemical education and scientific research although a quite different view of these concepts emerges from extremely successful application of quantum mechanical formalism to many chemical problems. This fact is intriguing and it is worth to take a closer look at the picture emerging from this physical theory.

In quantum mechanics, the atom is treated as a system of interacting particles - positively charged nucleus surrounded by negatively charged electrons. As a result, isolated atoms are electrically neutral. The state of such system and its evolution is described by respective state vector (wave function) obeying well known Schrödinger equation. The solution of this equation (with the assumption that we know the exact form of wave function) should in principle give us the entire knowledge about the system and its behavior. In practice it is not possible, since for every system of more than two particles (similarly as for famous three body problem formulated by Poincare), we cannot solve this equation analytically and the exact solution is not available (it is possible to obtain the analytical solution in case of hydrogen atom and singly ionized helium only). Almost all interesting systems, however, consists of heavier atoms or molecules and we are forced to make some approximations to make it possible to retrieve any useful information about the quantum system under study. We wish to focus on the two major approximations commonly used in computational physics and chemistry, namely adiabatic approximation (or Born-Oppenheimer approximation - the two names are often used interchangeably, despite small difference between these ap- 
proximations) and independent electrons approximation (self consistent field approach).

Since the forces exerted on electrons and nuclei constituting a molecule or a crystal, due to their electric charge are of the same order of magnitude, the resulting changes in their momenta must be the same. Consequently, it is rational to expect that the actual momenta of the electrons and nuclei are of a similar magnitude. Thus, since the nuclei are much heavier than the electrons, the electrons must move much faster than the nuclei. On a typical time-scale of the nuclear motion, the electron cloud will adjust instantly to changes in the nuclear configuration (they will relax to the instantaneous ground-state spatial configuration). As a result, the solution of the time-independent Schrödinger equation for the system of interest may rest on the assumption that the nuclei are stationary. In such a case one can solve this equation independently for the electronic ground-state first, then calculate the energy of the system in that configuration and at the end solve for the nuclear motion. Such separation of electronic and nuclear motion is known as the Born-Oppenheimer approximation.

The second approximation mentioned above, i.e. self consistent field approximation, is an approach to the problem of interacting electrons proposed by Hartree ${ }^{1}$, Fock $^{2}$ and Slater ${ }^{3}$. The electrons are treated independently of each other, moving in and interacting with self consistent electrostatic field taken as the spatial average over the positions of all other electrons. Thus the $\mathrm{N}$ electron problem is effectively reduced to much simpler one of solving $\mathrm{N}$ independent one-electron problems. While ignoring electron correlation effects, such an approach simplifies the N-electron problem considerably and allows us to perform the calculations for more complex systems. Unfortunately, the correlation effects are not negligible in many cases and additional steps including in some way

\footnotetext{
${ }^{1}$ D.R. Hartree, Proc. Cam. Phil. Soc., 24, 426 (1928).

${ }^{2}$ V. Fock, Z. Physik, 61, 126 (1930).

3 J.C. Slater, J. Chem. Phys., 1, 687 (1933).
} 
Andrzej Koleżyński

the electron correlation potential into the Hamiltonian are necessary. This led to the development of two different sets of computational methods. The first one consists of Heitler-London ${ }^{4}$ derivatives as a strong correlation limit and the second one incorporates various molecular orbital methods ( $a b$ initio Hartree-Fock and DFT Kohn-Sham ${ }^{5},{ }^{6}$ derivatives, where the correlation contribution can be included into the self consistent field).

Although the detailed analysis of this problem is beyond the scope of this study, it should be mentioned that, according to these two sets of methods, two different roads towards a qualitative as well as a quantitative understanding of a chemical bond have been pursued. Based on the concept of the resonance and the resonance structures and essentially using Heitler-London approach, Pauling proposed the Valence Bond (VB) method. The second road, the Molecular Orbital approach, was first developed by Hund and Mulliken and extended later among others by Slater, Lennard-Jones and $\mathrm{Hückel}^{7}$ and is basically founded on the assumption of independent electrons. When formulated broadly enough, both these roads, lead to identical result, but in practice only few lowest order corrections can be calculated for these two limiting cases. While MO method has been (and in fact still is) very successful, since most chemical bonds are relatively weekly correlated, it simply fails in cases with strong correlation effects (e.g. some metal-organic compounds). Nevertheless, regardless of the use of the MO or the VB approach, we are simply unable to restore the classical concept of chemical bond.

To sum up, physics furnishes us with the following picture: atoms are systems of positively charged nuclei, surrounded by interacting electrons. There is no such thing as an atom representing an individual entity with specific properties. It is rather a system

${ }^{4}$ W. Heitler and F. London, Zeitschrift für Physik, 44, 455 (1927).

${ }^{5}$ W. Kohn, L.J. Sham, Phys. Rev., 140, A1133 (1965).

${ }^{6}$ R.G. Parr, W. Yang, Density Functional Theory of Atoms and Molecules, Oxford University Press, New York 1989.

7 C. A. Coulson, Valence, Clarendon Press Oxford 1952. 
of interacting particles with the properties emerging from the interactions of its constituents. Cohesion and bonding are simple effects of the interactions among electrons and nuclei assigned to different atoms. The chemical bond is not an observable and so there is no chemical bonds as entities with well defined properties.

\section{The picture of atom and chemical bond in topological Quantum Theory of Atoms in Molecules}

Another type of approximations lead us to the concept of so called topological atom, defined within Bader's Quantum Theory of Atoms in Molecules ${ }^{8}$. Here we start with well defined physical quantum system and we calculate (irrespectively of the choice of the method) the total electron density distribution in system of interest (molecule or crystal). On such basis, a mathematical model of the chemical description of the matter that relies on the gradient dynamical system analysis within the framework of the Atoms in Molecules (AIM) theory is built up. The model is consistent with the postulates of quantum mechanics and valid for the exact wave functions and independent of the way of calculation of the latter or of chosen approximation. Bader has shown that the essence of the molecular structure of any system of interest (according to unequivocal partitioning of the physical space into regions assign to particular atoms) is contained in scalar field of its total electron density distribution. The main idea of Bader's theory is based on the theorem which states that to obtain well defined quantum-mechanical description of an open region of quantum system, this region has to be enclosed by the surfaces, obeying the relations $\Delta \rho(r) \cdot \mathbf{n}=0$ where $\mathbf{n}$ - is a vector of s a vector normal to the surface for every point of his surface (i.e. for which the gradient of the electron density scalar field is equal to zero - the so called zero-flux surfaces). Every such electron density distribu-

${ }^{8}$ R.F.W. Bader, Atoms in Molecules: A Quantum Theory, Oxford Univ Press, Oxford 1990. 
tion has very important topological property, namely the existence of maxima in atomic positions. Thus the above equation allows in practice the partitioning of the entire physical space of the system into adjacent regions where every region contains in principle only one maximum (atomic core). As a result of such partitioning, we are able to assign uniquely a part of physical space to individual atoms and restore the concept of atom in molecule or crystal (of course such ,atoms" do not have spherical symmetry like free atoms, but their symmetry is strictly connected with global symmetry of the system). Moreover, this approach yields useful information on the properties of the bonds and the chemical bonding in the system through the critical points that obey the condition $\Delta \rho(r)=0$. Thus the molecular structure becomes known as well.

There are four different types of critical points, but the most important for this particular analysis is the so called bond critical point (BCP), which indicates the existence of a chemical bond between given atoms. In general, every such bond critical point can be described by its position in physical space and respective electron density at this position, three eigenvalues $\lambda_{i}(i=1,2,3)$ of Hessian matrix of the electron density $\rho(\mathrm{r})$ and Laplacian $\Delta^{2} \rho(r)$ defined as a trace of Hessian matrix. All these parameters make up powerful tools allowing description and classification of a given structure: two negative eigenvalues of the Hessian matrix $\left(\lambda_{1}\right.$ and $\left.\lambda_{2}\right)$ measure the degree of contraction of electron density in directions perpendicular to each other and normal to the bond towards the BCP, while a positive eigenvalue $\left(\lambda_{3}\right)$ gives us a quantitative indication of the degree of contraction in direction parallel to the bond and from the BCP towards each of the neighboring nuclei.

The electronic charge is locally concentrated in the region of the BCP (covalent or polarized bonds) when negative eigenvalues dominate (this is characterized by large $\rho(r)$ values $\Delta^{2} \rho(r)<0$, and $\left|\lambda_{1}\right| / \lambda_{3}>1$. If positive eigenvalue dominates, the electronic density is locally concentrated at each atomic basin, the interaction is classified as closed shell and it is typical of highly ionic bonds, hy- 
drogen bonds, and van der Waals interactions (relatively low $\rho(\mathrm{r}$ ) values, $\Delta^{2} \rho(r)<0$ and $\left.\left|\lambda_{1}\right| / \lambda_{3}<1\right)$.

Moreover, the spatial distribution of the values of the function defined as negative of Laplacian of the total electron density (thus more positive values correspond to higher probability of finding of electron pair in given point in space) reveals the structure which, in case of molecules, is very similar to spatial molecular structure obtained within classical chemical model of VSEPR ${ }^{9}$ (Valence Shell Electron Pair Repulsion) - a direct descendant of classical model of covalent bonds formulated by Lewis ${ }^{10}$. From this very short and necessarily superficial description one can easily see, that such approach based on strict mathematical formalism allows us to restore to some extent the classical chemical picture of atoms (in molecules) and bonds.

The short description presented above allows us to draw conclusions on the evolution of the meaning of some quantum mechanical concepts. First of all, due to the approximations necessary to be assumed, in order to make chemical problems tractable within the framework of quantum mechanics we need to realize that the application of these numerous approximations makes quantum chemistry a distinct area of scientific inquiry in regards quantum mechanics. In other words, quantum mechanics is not directly applicable to chemical problems because the approximations introduced to this theory have changed the meaning of many fundamental quantum concepts within quantum chemistry. On the other hand, the enormous success of quantum chemistry strongly influences the view of many physicist and theoretical chemists on the ontology and properties of such entities like atoms or bonds. Moreover, this view contradicts that of classical chemistry so successful in the explanation of many crucial chemical problems. The approxima-

${ }^{9}$ N.V. Sidgwick, H.M. Powell, Proc. Roy. Soc., A 176, 153 (1940), R.J. Gillespie, R.S. Nyholm, Quart.Rev., 11, 339 (1957), R.J. Gillespie, J.Chem.Educ., 47, 18(1970)

${ }^{10}$ G. N. Lewis, J. Am. Chem. Soc., 38, 762 (1916). 
tions discussed here seem to show clearly the roots of a problem: we assume the separation of movements of nuclei and electrons as well as the independence of the movement of each electron; we apply these assumptions to the system under consideration and in many cases the obtained results are in a quite good agreement with experiment. The calculated values are used to draw the conclusions about the properties of atoms, molecules or bonds. At this point one needs to keep in mind that there is no such entity like a bond or a molecule in the original quantum mechanical description and the atom is definitely not the same entity as it is in quantum chemistry. Moreover, for many physicist and theoretical chemists this is sufficient evidence to deny the existence of a chemical bond! On the other hand, the latest development in theoretical chemistry, namely the topological analysis (Quantum Theory of Atoms in Molecules) is like Quantum Mechanics based on a strict mathematical formalism and in the same time it reveals the bonding structure of molecules. Compared with classical chemical view, it generates interesting questions about the ontological status of chemical bond, its nature and properties as well as its correspondence with the classical counterparts.

To sum up, it can be stated that some concepts defined within quantum mechanics evolve (and change their meaning) following the introduction of approximations that are necessary in the process of the implementation of the theory to practical problems in chemistry. This obviously seems to be unavoidable and in some way it is similar to the evolution of some physical concepts due to the development of physical theories. However, there is an important difference with respect to the evolution of concepts in physical theories. In physics such an evolution is usually carefully accounted for as these concepts are used in different theoretical context. In quantum chemistry, on the other hand, this difference is quite often underestimated or ignored despite of the simple fact that it can lead to serious misconceptions or false conclusions. Thus everyone carrying out theoretical analysis based on quantum chemical calcu- 
lations and drawing from their results some conclusions concerning chemical properties of the system of interest and/or composing it elements, has to be aware of the mentioned above problems and potential impact of chosen approximations on the final conclusions of such analysis when it is conducted in terms classical chemical concepts.

\section{Abstract}

The concepts of an atom and chemical bond in physics and chemistry: the role of approximations

It is well known from the history of science, that almost all crucial concepts are subject to change during the evolution of scientific theories. There is, however, another (often forgotten or omitted) aspect of this process, i.e. a role of some approximations, necessarily applied to any system of interest, in practically every single calculation carried out within quantum mechanical formalism, which can also result in change of the meaning of some of these concepts. In this paper, the two concepts of great importance in chemistry and physics, namely the concept of an atom, and the concept of a chemical bond are analyzed, both from the point of view of classical chemistry and quantum mechanics. The evolution of their meaning, due to the approximations, resulting in some important differences is highlighted.

Keywords: philosophy of chemistry, the concept of atom, the concept of chemical bond, evolution of meaning, quantum mechanics 